\title{
Morphology of galaxies with quiescent recent assembly history in a $\Lambda$-CDM universe
}

\author{
S. E. Pedrosa ${ }^{1,2}$, P. B. Tissera ${ }^{1,2,3}$, and M. E. De Rossi ${ }^{1,2,4}$ \\ ${ }^{1}$ Consejo Nacional de Investigaciones Científicas y Técnicas, CONICET, Argentina \\ e-mail: supe@iafe.uba.ar \\ 2 Instituto de Astronomía y Física del Espacio, Casilla de Correos 67, Suc. 28, 1428, Ciudad Autónoma de Buenos Aires, Argentina \\ 3 Departamento de Ciencias Fisicas, Universidad Andres Bello, Av. Republica 220, 4837-0134 Santiago, Chile \\ e-mail: patricia@iafe.uba.ar \\ ${ }^{4}$ Facultad de Ciencias Exactas y Naturales, Universidad de Buenos Aires, Ciudad Autónoma de Buenos Aires, \\ C1426EAI Buenos Aires, Argentina \\ e-mail: derossi@iafe.uba.ar
}

Received 18 November 2013 / Accepted 19 May 2014

\section{ABSTRACT}

\begin{abstract}
Context. The standard disc formation scenario postulates that the disc forms as the gas cools and flows into the centre of the dark matter halo, conserving the specific angular momentum. Major mergers have been shown to be able to destroy or highly perturb the disc components. More recently, the alignment of the material that is accreted to form the galaxy has been pointed out as a key ingredient to determine galaxy morphology. However, in a hierarchical scenario galaxy formation is a complex process that combines these processes and others in a non-linear way so that the origin of galaxy morphology remains to be fully understood.

Aims. We aim at exploring the differences in the formation histories of galaxies with a variety of morphologies, but quite recent merger histories, to identify which mechanisms are playing a major role. We analyse when minor mergers can be considered relevant to determine galaxy morphology. We also study the specific angular momentum content of the disc and central spheroidal components separately.

Methods. We used cosmological hydrodynamical simulations that include an effective, physically motivated supernova feedback that is able to regulate the star formation in haloes of different masses. We analysed the morphology and formation history of a sample of 15 galaxies of a cosmological simulation. We performed a spheroid-disc decomposition of the selected galaxies and their progenitor systems. The angular momentum orientation of the merging systems as well as their relative masses were estimated to analyse the role played by orientation and by minor mergers in the determination of the morphology.

Results. We found the discs to be formed by conserving the specific angular momentum in accordance with the classical disc formation model. The specific angular momentum of the stellar central spheroid correlates with the dark matter halo angular momentum and determines a power law. Our results suggest that gas accretion to rebuild the disc component is a necessary but not sufficient ingredient for a disc-dominated galaxy in a hierarchical clustering scenario. The survival of the discs is tightly linked to their subsequent history of accretion, as previously reported. Minor mergers play an important role. It is more likely that they will destroy the disc and feed the central spheroid if their angular momentum is misaligned with respect to that of the main galaxy. Conversely, if they are aligned, they can contribute to the disc formation.
\end{abstract}

Key words. galaxies: formation - galaxies: evolution - galaxies: structure

\section{Introduction}

The morphology of galaxies is the result of a complex combination of physical mechanisms acting at different scales and with different efficiencies. Observations show well-defined correlations between morphology and astrophysical properties such as star formation efficiency, metallicities, and sizes. Understanding these observations within a galaxy formation model is a challenging task. In the current cosmological paradigm, $\Lambda$-cold dark matter $(\Lambda-C D M)$, galaxy formation follows highly non-linear paths, involving the interplay of different physical processes, which finally determine the morphology of galaxies. Numerical simulations are a powerful tool to tackle such a complex problem. In the last decades, the development of more sophisticated sub-grid models for baryonic physics have contributed to simulate more realistic galaxies. Improved supernova $(\mathrm{SN})$ feedback schemes (Okamoto et al. 2005; Scannapieco et al. 2008; Agertz et al. 2011) together with a deeper understanding of the regulation of the star formation (Guedes et al. 2011; Agertz et al. 2011), and the action of SN outflows (Brook et al. 2011; Governato et al. 2012; Stinson et al. 2012) generate galaxies that better agree with observations. High numerical resolution simulations show that gas might flow into galaxies along filaments, feeding the galaxies directly with high angular momentum gas (Kereš et al. 2005; Dekel et al. 2009). However, even within this encouraging scenario it is still intensely debated how galaxies formed in dark matter haloes of different masses and which physical parameters are crucial in determining their final morphologies.

In the standard disc formation scenario (Fall \& Efstathiou 1980; Mo et al. 1998), the gas cools and flows toward the centre of the dark matter halo, conserving the specific angular momentum and forming rotational supported structures. Mergers have been shown to be able to easily destroy the discs and form spheroidal structures, principally during major mergers. First proposed by Toomre \& Toomre (1972), mergers have been 
considered as one of the main mechanisms responsible for the morphological evolution of the galaxy. However, major mergers have been shown to be rare in $\Lambda$ CDM (e.g. De Rossi et al. 2012), except for massive galaxies. Disc instabilities and minor collisions have also been claimed to be efficient processes to drive material from the disc towards the central regions (e.g. Barnes \& Hernquist 1996; Domínguez-Tenreiro et al. 1998; Tissera 2000; Mihos 2004; Scannapieco et al. 2009). Minor mergers have also been claimed to play an important role in the formation of elliptical galaxies at $z<2$ (e.g. Khochfar \& Silk 2006; Naab et al. 2009; Hopkins et al. 2010). Recently, observations and simulations suggest that the formation of stellar clumps in gasrich unstable discs might be another viable channel for classical bulge formation (e.g. Perez et al. 2013; Bournaud et al. 2014, and references therein).

Analysis of simulated galaxies with a variety of morphology suggests that alignment of the angular momentum of the accreted material with the angular momentum of the disc plays an important role in determining galaxy morphology (e.g. Scannapieco et al. 2009). Recently, Sales et al. (2012) reported the formation of disc-dominated galaxies to be correlated with coherent alignment of the angular momentum of baryons that are accreted over time. Martig et al. (2012) found that galaxies with the largest discs experienced no major mergers since $z \sim 2$ and had low and constant gas accretion histories, which keeps a stable angular momentum direction. These authors obtained disc-to-total $(\mathrm{D} / \mathrm{T})$ stellar mass ratios ranging from 0.4 to 0.8 for simulated galaxies in a cosmological simulation run with the RAMSES code (Teyssier 2002). Agertz et al. (2011) obtained Milky Way-like spirals with similar D/T values. Guedes et al. (2011) obtained a D/T fraction of 0.7 for Eris simulation, a very high-resolution simulation of a Milky Way-size galaxy (Diemand et al. 2008).

In this work, we aim at examining in more detail the evolution of the angular momentum content of galaxies with different morphologies at $z=0$, but with no recent major merger (1:3). It is well-known that major mergers have violent effects on the dynamics of discs and strongly affect them. Hence, the analysis of a sample of galaxies with no recent major merger but different final morphologies might contribute to obtain deeper insight into the role played in the angular momentum evolution by other physical processes such as minor mergers and the orientation of the accreted material.

Motivated by this reasoning, we analysed 15 galaxies that have quiescent recent history of evolution in the sense that none of them has experienced a major merger since $z \sim 3$. Our aim is to study the differences in their histories of formation and to understand the origin of their diverse morphology. For this purpose, we used a simulation from the Fenix Project to analyse the evolution of a galaxies with different morphologies. The analysed simulation represents a typical field region. The Fenix project aims at studying the chemical evolution of galaxies (Tissera et al., in prep.). Our sample is composed of 15 galaxies resolved with more than 20000 particles within the galaxy radius. These galaxies exhibit a variety of morphologies, with stellar mass D/T ratios varying from $\sim 0.3$ to 0.7 . These ratios are within the range found by previous works (Teyssier 2002; Diemand et al. 2008; Agertz et al. 2011). Nevertheless, there is a lack of systems with $\mathrm{D} / \mathrm{T}$ ratios close to unity (i.e. disc-dominated ones), which suggest an excess of stars formed in early stages of galaxy evolution (see discussion by De Rossi et al. 2013, regarding the star formation activity in this simulation). We study the angular momentum content, morphology evolution and the mergers events of the 15 chosen galaxies and their progenitor systems in detail.
This paper is organized as follows: in Sect. 2 we describe the numerical simulations. In Sect. 3 the main properties of the galaxy sample and their angular momentum content for the central spheroids, disc, and total baryonic masses are discussed. In Sect. 4, we analyse the evolution of morphology in relation to the properties of the accreted systems: angular momentum orientation and relative masses. In Sect. 5 we summarize our main results.

\section{Numerical experiments}

We analysed a cosmological simulation from the set of the Fenix project (S230A, Tissera et al. in prep.). This simulation has also been used by De Rossi et al. (2012) and De Rossi et al. (2010) to study the rotation curves and the Tully-Fisher relation of galaxies, and by De Rossi et al. (2013) to analyse the regulation of the star formation in haloes of different masses. The initial condition represents a typical field region of the $\Lambda$-CDM Universe with $\Omega_{\Lambda}=0.7, \Omega_{\mathrm{m}}=0.3, \Omega_{\mathrm{b}}=0.04$, a normalization of the power spectrum of $\sigma_{8}=0.9$ and $H_{0}=100 \mathrm{hm} \mathrm{s}^{-1} \mathrm{Mpc}^{-1}$, with $h=0.7$. A comoving cubic box of $14 \mathrm{Mpc}$ described with $2 \times 230^{3}$ initial particles, achieving a mass resolution of $5.9 \times 10^{6} h^{-1} M_{\odot}$ and $9.1 \times 10^{5} h^{-1} M_{\odot}$ for the dark matter and initial gas particles, respectively. The adopted gravitational softening length was $1.24 \mathrm{kpc} h^{-1}$. The minimum gas smoothing length was 0.10 , in units of the gravitational softening length. We used $\sim 33$ neighbouring particles for the hydrodynamics estimations.

The simulations were performed by using the code GADGET-3, an update of GADGET-2 (Springel \& Hernquist 2003; Springel 2005), optimized for massive parallel simulations of highly inhomogeneous systems. This version of GADGET-3 includes treatments for metal-dependent radiative cooling, stochastic star formation (SF), chemical enrichment, and the multiphase model for the interstellar medium (ISM) and the SN feedback scheme of Scannapieco et al. (2005, 2006). This $\mathrm{SN}$ feedback model is able to successfully trigger galactic massloaded winds without introducing mass-scale parameters, which makes it especially suitable for the study of galaxy formation in a cosmological context.

The multiphase model for the ISM allows the co-existence of diffuse and dense gas phases, as explained by Scannapieco et al. (2006). In this model, each gas particle defines its cold and hot phases by applying local entropy criteria, which allows gas particles to decouple hydrodynamically from particularly lowentropy particles, if they are not part of a shock front. Each coldgas particle stores the energy in a reservoir until they fulfil the conditions to be promoted and to join the hot phase. Our SN feedback scheme does not include free parameters that depend on the global properties of the given galaxy (such as the total mass and size), which makes it suitable for cosmological simulations where systems with different masses form in a complex way.

The chemical model describes the enrichment by Type II (SNII) and Type Ia (SNIa) supernovae according to the nucleosynthesis productions of Woosley \& Weaver (1995) and Thielemann et al. (1993). All stars more massive than $8 M_{\odot}$ are assumed to end their lives as SNII, while for the rate of SNIa, an observationally motivated value of 0.0015 is adopted (Mosconi et al. 2001). To estimate the number of stars of a given mass, we assumed a Salpeter initial mass function. The time-delay for the ejection of material of SNIa is randomly selected within the range of $[0.1,1] \mathrm{Gyr}$, while SNII are assumed to explode within the integration time step. The energy released 
by each $\mathrm{SN}$ event $\left(0.7 \times 10^{51} \mathrm{erg} \mathrm{s}^{-1}\right)$ is distributed into the cold and hot gas media surrounding a star particle. A fraction $\epsilon_{\mathrm{c}}$ of the energy released by each $\mathrm{SN}$ event is injected into the cold gaseous medium, while the rest is pumped into the hot phase. For $\mathrm{S} 230 \mathrm{~A} \epsilon_{\mathrm{c}}=0.5$, chemical elements are distributed using the same $\epsilon_{\mathrm{c}}$, but are injected into the cold and hot phase as soon as they are synthesized.

\section{Main galaxies}

We study in detail the main galaxies in virialized haloes identified by using a friends-of-friend and the SUBFIND algorithm (Springel et al. 2001). A main galaxy is defined as the more massive system within a virial potential well. The physical properties for the main galaxies are calculated within the galaxy radius $\left(r_{\text {gal }}\right)$ defined as the one that enclosed $\sim 80$ percent of the baryonic mass of a galaxy. Simulated galaxies with more than 2000 total particles within $r_{\text {gal }}$ in S230A have also been analysed by De Rossi et al. (2010), who found them to reproduce the observed baryonic Tully-Fisher relation. At $z=0$, the star formation efficiencies reproduce observed motivated trends (Moster et al. 2013), as reported by De Rossi et al. (2013). However, as pointed out by these authors, these simulated galaxies still show an excess of stars, which can be tracked to a highly efficient transformation of gas into stars at high redshift. This is an open problem, although there are results that suggest that the early action of stellar feedback from massive stars (Stinson et al. 2012; Aumer et al. 2013) or even high-energy sources such as X-ray high-mass binaries (Power et al. 2013; Artale et al. 2013) might help to regulate the star formation activity at very high redshift. Even with this caveat in mind, it is possible to learn about galaxy formation by analysing the differences in the final morphologies in relation to the formation history of the galaxies (e.g. Scannapieco et al. 2009; Martig et al. 2012; Sales et al. 2012).

To reduce numerical resolution problems, we restricted our analysis to the main galaxies resolved with more than 20000 baryons within $r_{\text {gal }}$. This criterion yielded a subsample of 15 main galaxies (hereafter, the 15 chosen galaxies), with virial masses ranging from $\sim 3 \times 10^{11} M_{\odot}$ to $\sim 3 \times 10^{12} M_{\odot}$, all of them resolved with $\sim 100000$ to $\sim 900000$ total particles. For a fully cosmological simulation, these haloes are quite well resolved and allow us to analyse the properties of the galaxies (see also Torrey et al. 2014). We also know from previous analysis of a higher resolution simulation, with a factor of eight in particle number and the same physical model, that the properties of galaxies are well represented, as discussed in detail by De Rossi et al. $(2012,2013)$.

By adopting the criteria proposed by Tissera et al. (2012), we decomposed the stellar populations into three dynamical components: central spheroid, disc, and stellar halo. For this purpose, we estimated the parameter $\epsilon$ of the star (gas) particles defined as $\epsilon=j_{z} / j_{z \text {, max }}(E)$, where $j_{z}$ is the angular momentum component in the direction of the total angular momentum, and $j_{z, \max }(E)$ is the maximum $J_{z}$ over all particles of given binding energy, $E$. A particle on a perfect prograde circular orbit in the disc plane has $\epsilon=1$. We considered particles with $\epsilon$ higher than 0.5 to be part of a disc. This is a low threshold, but it is consistent with the gravitational length used to run these simulations $\left(\sim 1.2 \mathrm{kpc}^{-1}\right)$. The particles that did not satisfy this requirement were taken to belong to the spheroidal component. By inspecting the $\epsilon-E$ plane, we checked wether the adopted limits were suitable to individualize the rotationally supported components. We also distinguished between the central spheroid and
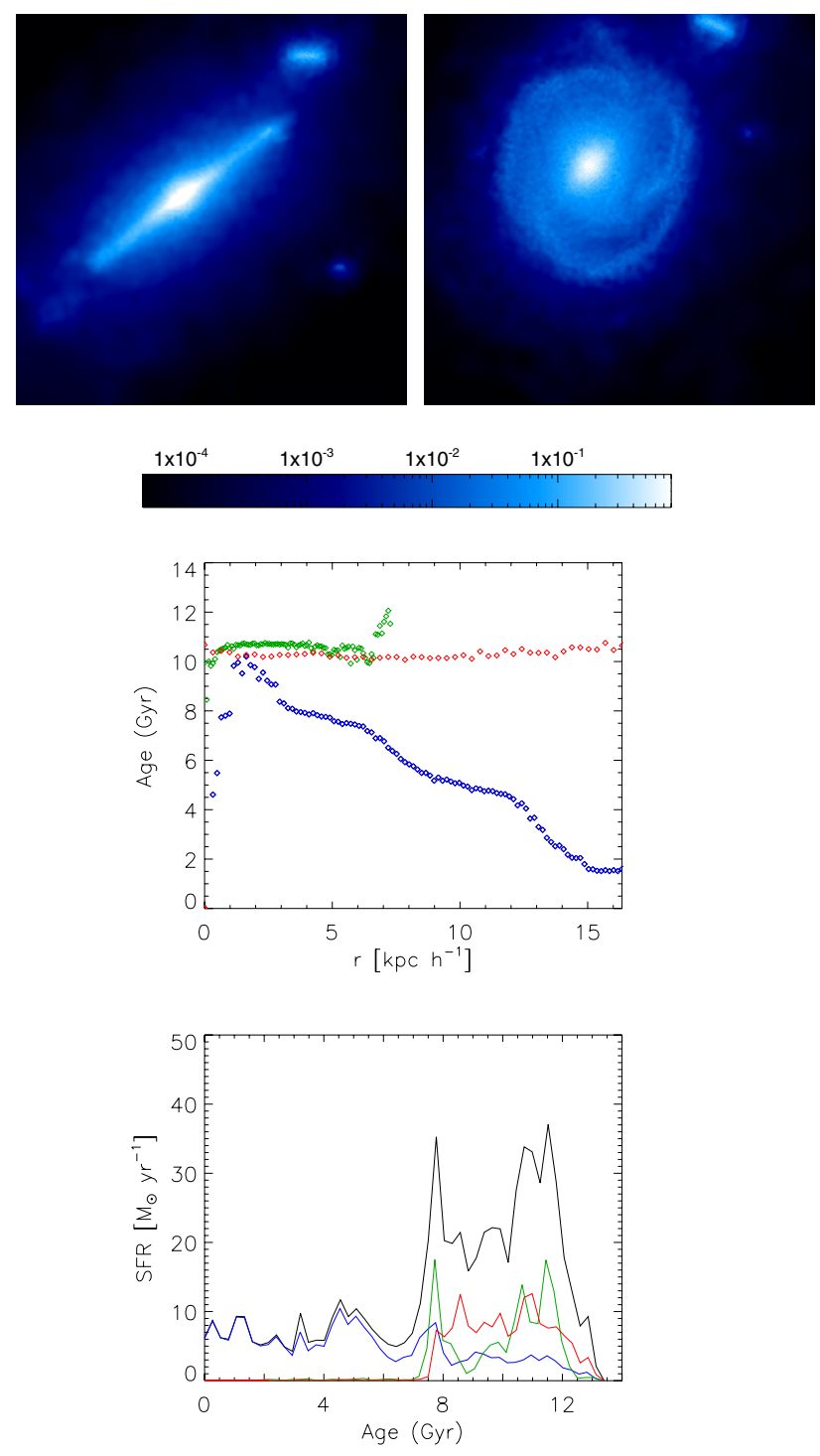

Fig. 1. Edge-on and face-on density maps for a typical galaxy of our catalogue (G1 in Table 1) in simulation S230A (upper panels). The color bar shows stellar density in units of $10^{10} M_{\odot} \mathrm{kpc}^{-3}$. The middle and lower panels show the age gradients and star formation rate for stars in the central spheroid (green), the disc (blue), the stellar halo (red). The solid black line in the lower panel correspond to the total star formation rate.

the stellar halo components. The central spheroid is composed of the most bounded particles, while the less bounded ones are considered to form the stellar halo. Following Tissera et al. (2012), we adopted the minimun $E$ value of the particles that fill a casket at half of $r_{\text {gal }}$ to distinguish the central spheroid from the stellar halo. Particles less bounded than these were considered to be part of the halo. This energy-based criterium was successfully used with the higher resolution simulations of the Aquarius galaxies (Tissera et al. 2012, 2013). Nevertheless, note that the stellar haloes do not contribute largely to the mass in the central parts that are dominated by the most bounded particles. Hence, appreciable variations of these criteria do not affect our estimations.

In Fig. 1 we show the face-on and edge-on projected stellar density maps for galaxy G1 in Table 1, which has a large stellar disc at $z=0$. In the lower panel we show the age profiles for the different stellar components as a function of radius. It can be appreciated that stars in the disc component determine a 
Table 1. Main characteristics of our subsample of the 15 most massive objects in S230A.

\begin{tabular}{|c|c|c|c|c|c|c|c|c|c|c|}
\hline Galaxy & $N_{\text {dark }}$ & $N_{\text {star }}$ & $N_{\text {gas }}$ & $\begin{array}{c}R_{200} \\
\left(\mathrm{kpc} h^{-1}\right)\end{array}$ & $\begin{array}{c}M_{200} \\
\left(10^{11} M_{\odot} h^{-1}\right)\end{array}$ & $\begin{array}{c}M_{\mathrm{star}} \\
\left(10^{10} M_{\odot} h^{-1}\right)\end{array}$ & $\begin{array}{c}M_{\mathrm{bar}} \\
\left(10^{10} M_{\odot} h^{-1}\right)\end{array}$ & $n$ & $\begin{array}{c}R_{\mathrm{d}} \\
\left(\operatorname{kpc} h^{-1}\right)\end{array}$ & $D / T$ \\
\hline G1 & 437013 & 255241 & 192495 & 231.1 & 25.9 & 7.7 & 8.0 & 1.1 & 4.1 & 0.56 \\
\hline G2 & 156183 & 92670 & 28190 & 162.2 & 9.3 & 3.1 & 3.3 & 2.2 & 2.5 & 0.55 \\
\hline G3 & 228052 & 120262 & 82041 & 185.2 & 13.5 & 4.0 & 4.3 & 1.5 & 3.2 & 0.46 \\
\hline G4 & 162563 & 72102 & 44930 & 164.6 & 9.6 & 2.3 & 2.4 & 1.4 & 2.1 & 0.71 \\
\hline G5 & 71815 & 48399 & 14528 & 125.5 & 4.3 & 1.8 & 1.8 & 1.3 & 1.5 & 0.34 \\
\hline G6 & 181662 & 113855 & 52922 & 171.6 & 10.8 & 3.9 & 4.3 & 1.9 & 3.1 & 0.56 \\
\hline G7 & 117001 & 60969 & 49564 & 148.6 & 6.9 & 2.1 & 2.4 & 1.3 & 5.1 & 0.44 \\
\hline G8 & 82096 & 40720 & 23221 & 131.1 & 4.9 & 1.4 & 1.7 & 1.3 & 5.6 & 0.39 \\
\hline G9 & 69753 & 31088 & 15567 & 123.8 & 4.1 & 1.1 & 1.5 & 1.3 & 4.0 & 0.52 \\
\hline G10 & 260771 & 154477 & 74724 & 193.2 & 15.5 & 5.0 & 5.3 & 1.3 & 4.2 & 0.47 \\
\hline G11 & 136695 & 71156 & 28907 & 155.1 & 8.1 & 2.4 & 2.5 & 1.4 & 1.9 & 0.59 \\
\hline G12 & 117048 & 49347 & 27641 & 147.1 & 6.9 & 1.4 & 1.5 & 1.1 & 2.8 & 0.50 \\
\hline G13 & 129747 & 71671 & 36577 & 153.0 & 7.7 & 2.6 & 2.7 & 1.5 & 1.7 & 0.66 \\
\hline G14 & 62344 & 27559 & 14604 & 119.2 & 3.7 & 0.9 & 1.0 & 1.3 & 3.5 & 0.49 \\
\hline G15 & 49406 & 24659 & 7370 & 110.0 & 2.9 & 0.8 & 0.9 & 1.3 & 1.4 & 0.27 \\
\hline
\end{tabular}

Notes. Columns: number of dark matter $\left(N_{\text {dark }}\right)$, star $\left(N_{\text {star }}\right)$, and gas $\left(N_{\text {gas }}\right)$ particles within the virial radius, virial radius $\left(R_{200}\right)$, virial mass $\left(M_{200}\right)$, stellar $\left(M_{\mathrm{star}}\right)$ and baryonic $\left(M_{\mathrm{bar}}\right)$ masses within the baryonic radius, Sérsic index for the central spheroid $(n)$, disc scale length $\left(R_{\mathrm{d}}\right)$, and disc-tototal-mass ratio $(\mathrm{D} / \mathrm{T})$.

well-defined negative age gradient consistent with an inside-out history of formation. This is a common feature of our simulated galaxies that were able to retain large stellar discs. Conversely, the central spheroid and halo stellar components do not show a clear age profile and are populated by old stars with mean ages of $\approx 10$ Gyr. Stars in the central spheroids are systematically older than those in the stellar haloes. This reflects their different assembly history: the central spheroids are mainly formed in situ, within the deepest potential wells, while the stellar haloes are principally assembled from small substructures (Tissera et al. 2012). Even though the SFRs of the central spheroid and stellar halo show the highest activity at very early times, the former transformed most of their stars in a shorter burst. For the system shown in Fig. 1, the stars in the central spheroid were mainly formed between $\sim 10$ and $\sim 12$ Gyr ago and with a rejuvenating short-lived peak at $\sim 8$ Gyr. The stellar halo exhibits a more extended star formation history from $\sim 12$ to $\sim 8$ Gyr ago. As expected, the disc component experiences the higher star formation activity at low and intermediate redshift with some contribution from old stars. For this system, the mean age of the stellar populations in the disc is $\sim 5 \mathrm{Gyr}$ and the contribution of stars with ages older than 8 Gyr represents $\sim 23$ per cent of the total stellar mass. The inside-out disc formation scenario for normal disc galaxies has been confirmed by several $N$-body/SPH simulations (e.g. Guedes et al. 2011; Agertz et al. 2011). The standard disc formation scenario (see e.g. Mo et al. 1998) provides a physical explanation for observational results where negativeage gradients are commonly found. In particular, recent detailed observations of the CALIFA survey reported disc components to have negative-age gradients and bulges to be consistent with older populations with flatter profiles in spiral galaxies with total stellar masses larger than $10^{11} M_{\odot}$ (Pérez et al. 2013; see also MacArthur et al. 2004).

We performed a double component decomposition for each of the 15 chosen galaxies. For the discs, we adopted an exponential profile with a scale-length $R_{\mathrm{d}}$. For the central spheroid, we assumed a Sérsic law (Sérsic 1963) with an index $n$ and a scale-length $R_{\mathrm{b}}$. As can be seen in Table 1, we found a Sérsic index lower than $n \sim 2$, consistent with pseudo-bulges. Numerical results reported by Martig et al. (2012) were similar and seem to agree with observational findings of local disc galaxies where mean $n$ values lower than two are reported (Laurikainen et al. 2007; Weinzirl et al. 2009). In Table 1, we also include the D/T fraction defined as $M_{\text {disc }} /\left(M_{\text {disc }}+M_{\text {spheroid }}\right)$, where $M_{\text {disc }}$ and $M_{\text {spheroid }}$ are the dynamical masses of the disc and central spheroidal components obtained by the kinematic decomposition described above.

We used the recent observational results from Reyes et al. $(2011,2012)$ to compare the size-stellar mass relation determined by our simulated disc galaxies. Because the authors estimated the optical radius $R_{80}$ for their observed galaxies, we defined $r_{80}$ as the radius that encloses $\sim 80$ of the stellar mass of a given galaxy. In doing so, we asumed a constant mass-tolight ratio (Tissera 2000) In Fig. 2, we plot $r_{80}$ as a function of the galaxy stellar mass for the simulated galaxies and the observed values reported by Reyes et al. (2011). The simulated galaxy sizes generally agree with these observations, although they tend to populate the upper part of the observed distribution because of the action of the strong SN feedback adopted in this run, which produces more extended baryon distributions ${ }^{1}$. Unfortunately, the high numerical resolution limit imposed on the selected galaxies left us with a low number sample to assess the existence of a correlation between galaxy scale and galaxy mass. This price is worth paying because we aim at analysing the evolution of the angular momentum across time, which can be easily affected by a low number of particles.

\subsection{Angular momentum content}

The angular content of the galaxies in relation to that of their host dark matter haloes has been analysed in previous works, as discussed in the Introduction. It has been shown that the disc components tend to form by conserving the specific angular momentum as postulated by the standard disc formation model.

\footnotetext{
1 Scannapieco et al. (2006) discussed in detail the effects of the SN feedback model we use in S230 on baryons by running the same halo with different SN feedback parameters. In addition, in De Rossi et al. (2010) we compared S230 with a run where the SN feedback was switched off. These analyses show that only when SN feedback was on, extended discs were produced.
} 


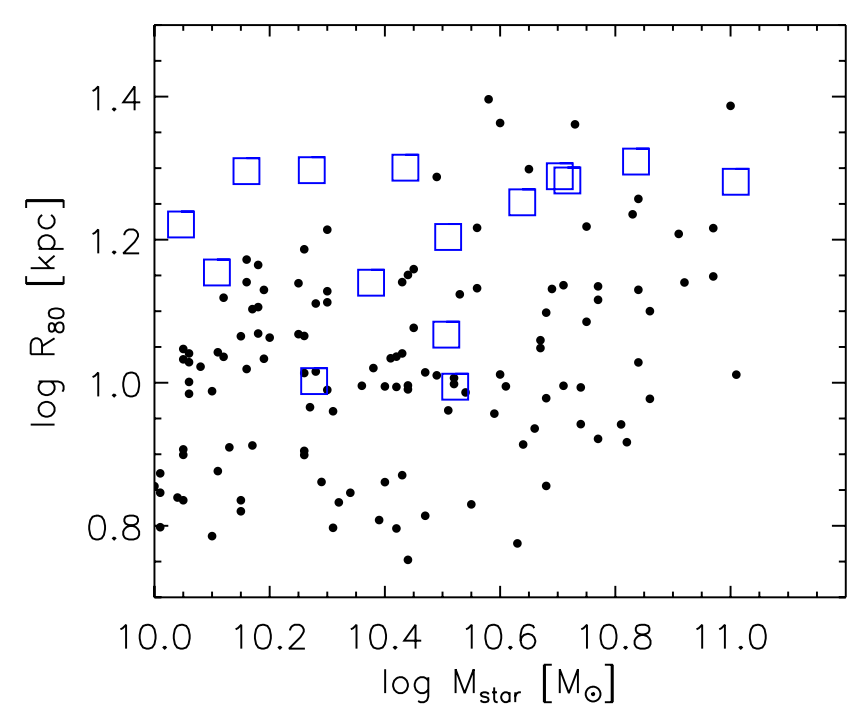

Fig. 2. Optical radius as a function of the stellar mass of the simulated galaxies (blue open squares) and the observational estimates of Reyes et al. (2011, black circles).

In this work, we extend this analysis by examining the angular momentum content of the disc as well as of the central spheroidal components in galaxies with different $\mathrm{D} / \mathrm{T}$ ratios.

We estimate the specific angular momentum of the stellar and gaseous disc components within $r_{\text {gal }}$ and of the dark matter haloes within $R_{200}$. In the model of Mo et al. (1998), it is assumed that the mass that settles into the disc is a fixed fraction of the dark matter halo mass, $m_{\mathrm{d}}=M_{\mathrm{D}} / M_{\mathrm{H}}$. Similarly, its angular momentum is assumed to be a fraction $j_{\mathrm{d}}$ of that of the halo defined as $j_{\mathrm{d}}=J_{\mathrm{D}} / J_{\mathrm{H}}$. Following these concepts, we calculated $m_{\mathrm{d}}$ and $j_{\mathrm{d}}$ for the stellar and gaseous discs. We also applied these definition for the total baryonic mass within $r_{\mathrm{gal}}$.

As can be seen in Fig. 3, we found a clear correlation between the angular momentum of both gaseous and stellar discs and that of the haloes, with the $j_{\mathrm{d}} \propto m_{\mathrm{D}}$ relation as expected for angular momentum conservation. Under the hypothesis of angular momentum conservation, $j_{\mathrm{d}}$ should be equal to $m_{\mathrm{d}}$ (Fall \& Efstathiou 1980). This assumption is based on the fact that all the material in the system experiences the same external torques before separating into two distinct components. The stellar discs show the best behaviour, while the gaseous discs have gained additional angular momentum because of the action of the SN feedback, which generates more extended gas distributions. On average, the 15 chosen galaxies in S230A have stellar discs that agree with the hypothesis of angular momentum conservation.

In Fig. 3, we also show $j_{\mathrm{s}}$ versus $m_{\mathrm{s}}$ estimated by using stellar masses of the central spheroids. As can be seen from this figure, these stars have been formed from low angular momentum material. Nevertheless, there is a clear correlation between the specific angular momentum of the total stellar mass of the central spheroids and their dark matter haloes so that $j_{\mathrm{s}} \propto m_{\mathrm{s}}^{0.7}$. The correlation is noisier than that determined by the discs, but is still significant $(r \sim 0.6)$. The angular momentum content of the stellar components in both the central spheroids and the disc components can be affected by the mergers and interactions (e.g. if there is angular momentum transfer between the components and the halo) and by the SN feedback, which helps to regulate the star formation activity, but also can affect the dynamical evolution of the gas from which the next generation of stars will form.

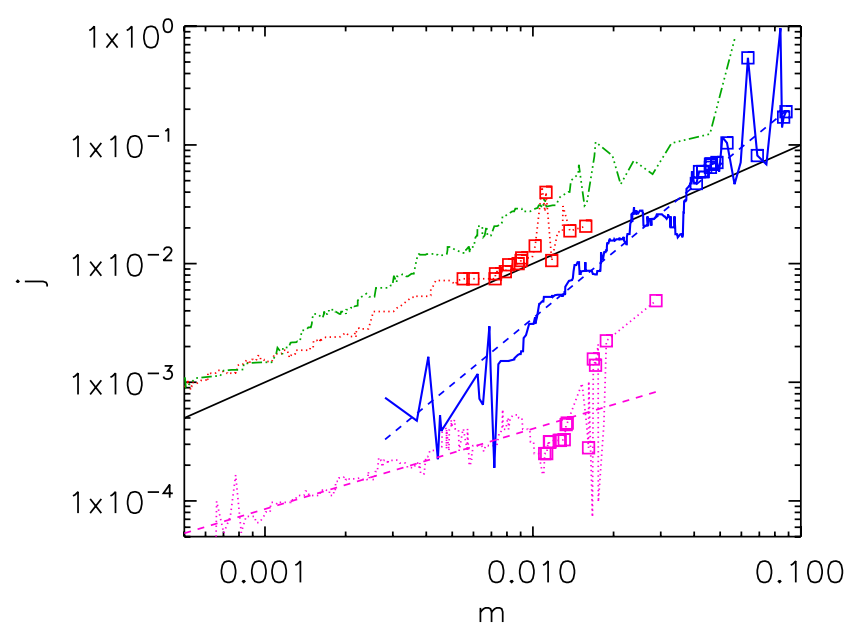

Fig. 3. Median angular momentum fraction $\left(J_{\mathrm{D}} / J_{\mathrm{H}}=j_{\mathrm{d}}\right)$ for the stellar (dotted red line) and gaseous (dash-dotted green line) disc components as a function of the ratio between the stellar and gaseous disc masses and of the dark matter haloes. We also show the same relation for the stellar central spheroids (dashed pink line), which determines a welldefined correlation, but still consistent with weaker angular momentum conservation. The relation for the total baryonic mass is also included (solid blue line). The dashed blue line shows the relation $j \propto \mathrm{m}^{2}$. The dashed pink line represents the linear fit to the relation defined by stellar central spheroids.

The specific angular momentum of the total baryonic component of a galaxy and that of its dark matter halo determine a relation close to $j_{\text {bar }} \propto m_{\text {bar }}^{2}$. This trend agrees with the results reported by Sales et al. (2012), although the SN feedback models are different. The change in the slope relation in our simulated galaxies may be due to the effects of the redistribution of angular momentum between the components during violent events (e.g. Pedrosa et al. 2010, and references therein), combined with the action of SN feedback that transfers angular momentum to the gas component and drives powerful winds. It is expected that these winds remove preferentially low angular momentum gas (e.g. Brook et al. 2011).

Our results show that if a disc component is able to form, it does so according to the classic formation scenario via conservation of the specific angular momentum, in agreement with previous works. The material that forms the disc conserved the specific angular momentum obtained via tidal torques by the initial density perturbations. We also find that stellar central spheroids, which formed from low angular momentum material, determine a clear correlation with the angular momentum content in the dark matter halo. These results might contribute to explain the observational size-mass relation reported by Kravtsov (2013). As suggested by this author, the angular momentum content might play a critical role in setting the size of galaxies of different morphologies, even spheroidal-dominated ones. A comprehensive analysis of the angular momentum evolution of the dynamical components and the relation with the sizes will be performed in forthcoming paper (Pedrosa \& Tissera, in prep.).

\section{Formation and survival of the stellar discs}

We quantified the evolution of the morphology by analysing the $\mathrm{D} / \mathrm{T}$ ratios as a function of redshift and the correlation of this parameter with the assembly history of the simulated galaxies. As galaxies evolve, the $\mathrm{D} / \mathrm{T}$ ratios of the progenitor systems can change, reflecting their different assembly histories. We adopted 


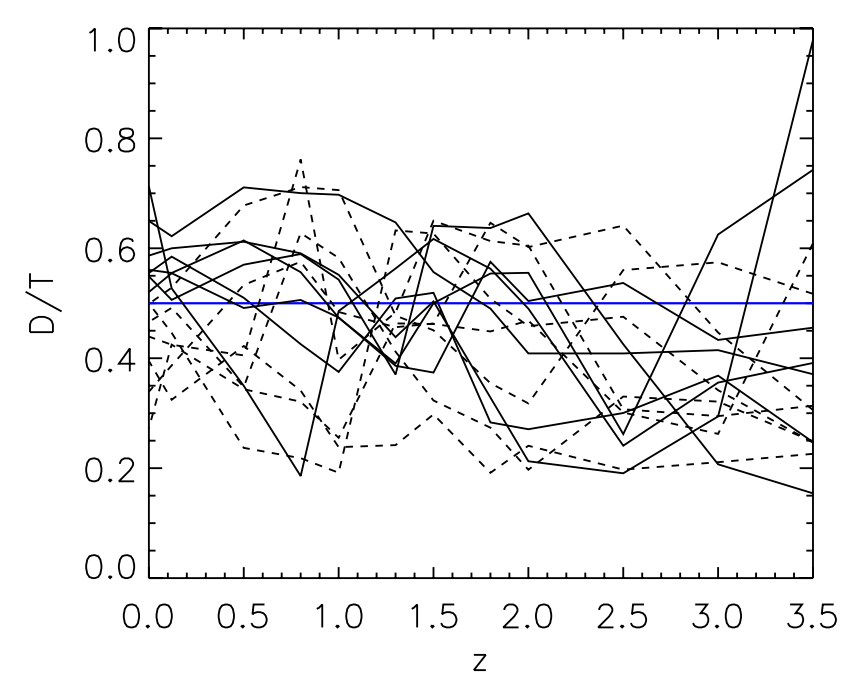

Fig. 4. D/T ratios for the progenitor galaxies of the 15 chosen galaxies as a function of the redshift. Those with $D / T_{z=0}>0.5$ are shown by continuous lines and those with $D / T_{z=0}<0.5$ by dashed lines.

$D / T=0.5$ as a threshold to separate our simulated galaxies into two groups: disc and spheroidal-dominated systems. At $z=$ 0 , the 15 chosen galaxies have $\mathrm{D} / \mathrm{T}$ values ranging from $\sim 0.3$ to $\sim 0.7$.

We constructed the merger tree for each simulated galaxy at $z=0$ and followed the evolution of the main progenitor branch. At each available cosmic time, we defined the main progenitor as that which exhibits the most significant baryonic component. All other smaller systems in the merger tree were considered satellites that will eventually merge onto the main branch (see also De Rossi et al. 2012).

To examine the evolution of the $\mathrm{D} / \mathrm{T}$ ratios, we performed the kinematical decomposition explained in Sect. 3 to the progenitor systems of each selected galaxy up to $z \sim 3.5$. For the purpose of illustration, we show their evolution with redshift in Fig. 4. It is clear that the $\mathrm{D} / \mathrm{T}$ ratio is a very dynamic parameter, which indicates that the disc can be destroyed and re-built several times along its evolution. Our goal is to try to underpin the physical conditions or processes that determine these behaviours in relation to the final morphologies.

As discussed in the Introduction, none of the 15 chosen galaxies has experienced a major merger (1:3) since $z \sim 3$, so they might be considered to have quite recent assembly histories. Nevertheless, they all end with different $\mathrm{D} / \mathrm{T}$ ratios. Hence, the differences in the final morphology have to respond to their different assembly histories.

We explored the correlation between the $D / T_{z=0}$ and those of the progenitor systems measured at $z=0.5, z=1$ and $z=2$. As shown in Fig. 5, there is a global trend for the simulated galaxies to have their morphology in place since $z \sim 1$. However, by $z \sim 2$ the correlation is lost, suggesting that, at least in this simulation, the morphology of the final galaxies is principally determined by their history at $z>1$, and for lower redshift, the morphologies of these simulated disc galaxies are not strongly modified, on average. We note that on an individual basis, there might be different behaviours, as can be seen from the large dispersion shown by the relations. The correlation factors are $0.29,0.43$, and -0.1 for relations measured at $z=0.5, z=1$, and $z=2$, respectively. On average, the evolution since $z \sim 1$ does not strongly affect the morphology of the simulated galaxies. By using a different numerical approach, Martig et al. (2012) found no correlation between the morphology at $z=0$ and at $z=1$. This difference could be due to the different numerical implementations or to the different set of analysed galaxies since in our work.

A crucial ingredient for disc formation is the availability of accreted gas needed to rebuild the disc, as shown in previous works (Sales et al. 2012). We estimated the growth of $M_{\text {disc }}$ and $M_{\text {spheroid }}$ from $z \sim 3.5$ to $z=0$. We found that galaxies with $D / T_{z=0}>0.5$ have mean $M_{\text {disc }}$ larger or similar to the mean $M_{\text {spheroid }}$ since $z \sim 2$, while those with $D / T_{z=0}<0.5$ have central spheroids more massive than the discs in the same redshift range. For galaxies with $D / T_{z=0}>0.5$, we estimated that the disc components have grown by a factor $\sim 2.5$ since $z \sim 1$ while the discs in spheroid-dominated galaxies have done so only by $\sim 1.2$. The stellar masses in the central spheroids have grown by a similar factor $(\sim 1.5)$ for all galaxies, implying that the disc components have grown more in disc-dominated galaxies, as expected. The relative increase of the disc-to-centralspheroid mass is due to the gas availability and the fact that the discs are able to survive and grow. For spheroidal-dominated galaxies, the growth of the central spheroid is larger than that the of the disc. In next section, we analyse how minor merger events can be associated to the decrease of the $\mathrm{D} / \mathrm{T}$ ratio.

The estimation of the redshift at which 50 percent of the final stellar mass in the discs was at place yielded that all discs in our sample have built up half their stellar masses at $z<2$. We detected a weak correlation between these parameters, suggesting that systems that formed their discs earlier have a higher $\mathrm{D} / \mathrm{T}$ ratio at $z=0$.

\subsection{Galaxy morphology evolution}

We followed the formation history of galaxies and studied their angular momentum content, their growth of the discs and central spheroidal components, and their merger history. We estimated the stellar masses of the progenitor galaxies $\left(M_{\mathrm{gal}}\right)$ and of accreted satellites $\left(M_{\mathrm{sat}}\right)$, the angle between the total angular momenta of progenitor galaxy $\left(J_{\mathrm{gal}}\right)$ and of accreted satellites $\left(J_{\mathrm{sat}}\right)$ at each available snapshot of the simulation since $z \sim 3.5$. As galaxies evolve across time, $\mathrm{D} / \mathrm{T}$ experiences strong variations that might be linked with interactions with the satellites that merge with the progenitor galaxy. We defined $\Delta_{\text {fdisc }}$ as the variation of the $\mathrm{D} / \mathrm{T}$ associated to the main progenitor of a given galaxy between two consecutive snapshots in the simulation, where the second snapshot is the one where the satellite can no longer be identified as a separate object ${ }^{2}$. Hence, $\Delta_{\text {fdisc }}>0$ implies a positive growth of the stellar disc component with respect to the stellar central spheroid between the two available snapshots. In Fig. 6, we plot $\Delta_{\text {fdisc }}$ as a function of the ratio $M_{\mathrm{gal}} / M_{\mathrm{sat}}$. As can be seen, major mergers (1:3) are all associated to negative changes of $\mathrm{D} / \mathrm{T}$, which indicates that the central spheroids grow with respect to the discs during these events. When less massive mergers are considered, the response is different, with positive and negative variations.

We explored the impact of the relative orientation between the angular momenta of the progenitor galaxies and the accreted satellites by assuming that the interaction is aligned when $\cos \left(J_{\text {gal }}, J_{\text {sat }}\right)$ is larger than 0.7 and misaligned when, $\cos \left(J_{\text {gal }}, J_{\text {sat }}\right)<-0.7$ or $-0.3>\cos \left(J_{\text {gal }}, J_{\text {sat }}\right)<0.3$. We separated these extreme behaviours to highlight the effects more

\footnotetext{
2 Since the number of available snapshots is limited, the exact time of a merger might not be available. This temporal limitation would contribute to increase the noise in the relations. Hence, only averaged trends can provide useful information.
} 
S. E. Pedrosa et al.: Morphology of galaxies
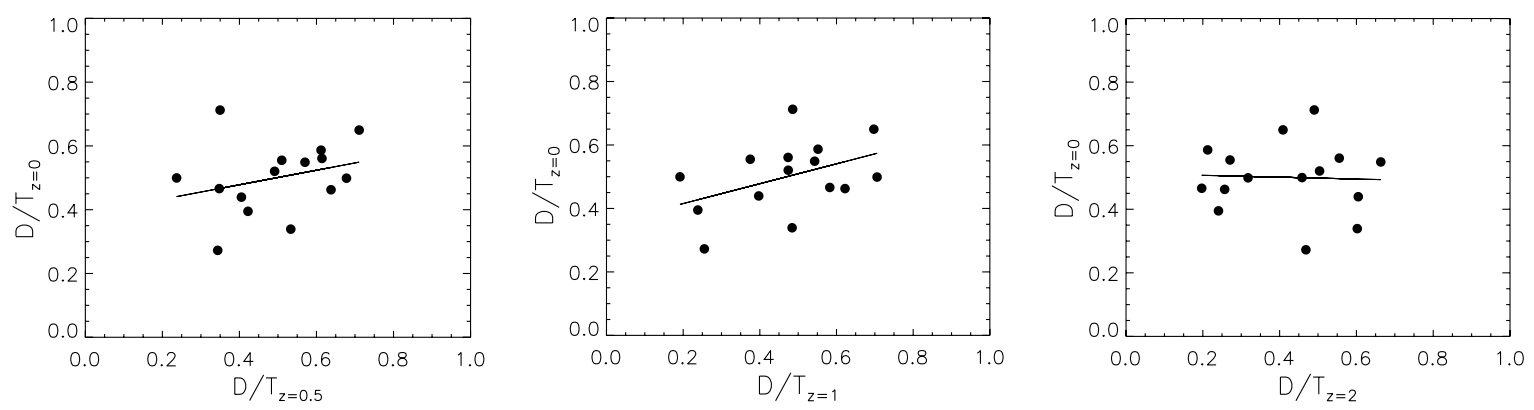

Fig. 5. Correlation between the $\mathrm{D} / \mathrm{T}$ ratios of the simulated massive galaxies in $\mathrm{S} 230 \mathrm{~A}$ at $z=0$ and those of their progenitor systems at $z=0.5,1$ and $z=2$. The solid lines are regression fits to the data.

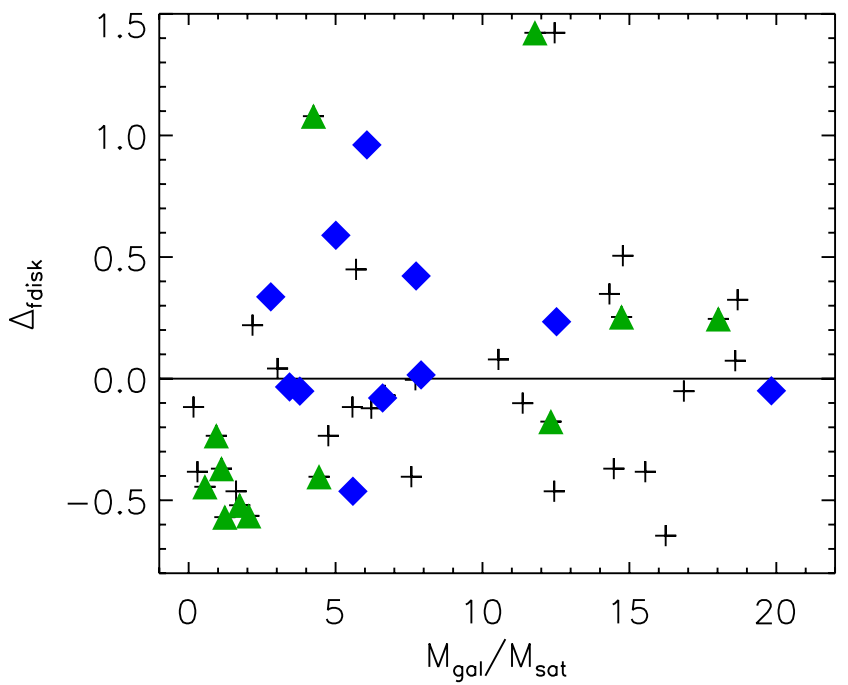

Fig. 6. $\Delta_{\text {fdisc }}$ as a function of the $M_{\text {gal }} / M_{\text {sat }}$ for the colliding systems. We show the aligned (blue diamonds) and misaligned (green triangles) encounters. Aligned encounters are systematically associated to positive or negligible changes of the $\mathrm{D} / \mathrm{T}$. Crosses: encounters without defined alignment.

clearly. The interactions that cannot be classified within these categories are shown as crosses in Fig. 6. It is clear that aligned interactions produce negligible or positive variations of $\mathrm{D} / \mathrm{T}$. The median $\Delta_{\text {fdisc }}$ estimated for aligned mergers is $\Delta_{\text {fdisc }} \sim 0.015$, for the misaligned mergers this is $\Delta_{\text {fdisc }} \sim-0.44$. We note that more than $60 \%$ of the encounters that are not aligned, but have not entered our stringent misalignment classification, are associated with negative changes in the $\mathrm{D} / \mathrm{T}$ ratio.

Regarding the relative masses of the interacting systems, we also found that encounters with relative stellar masses up to $M_{\mathrm{gal}} / M_{\mathrm{sat}} \sim 6$ can have a strong impact on the galaxy morphology if they are misaligned. Accretions of satellites that are aligned with the angular momentum of the progenitor galaxy have a negligible impact or even increase the disc components, regardless of $M_{\mathrm{gal}} / M_{\mathrm{sat}}$, on average. We found misaligned minor and major mergers to be associated with a strong perturbation of the disc components. This result agrees with previous works (e.g. Domínguez-Tenreiro et al. 1998; Scannapieco et al. 2009), who showed that misaligned accretions can be correlated with the disc destruction, and with the global conclusions of Sales et al. (2012), who found a correlation with the total angular momentum content at turnaround of the accreted material. Considering the results of Sales et al. (2012), we would expect that the galaxies that have large discs at $z=0$ would have experienced more aligned mergers during their assembly history.

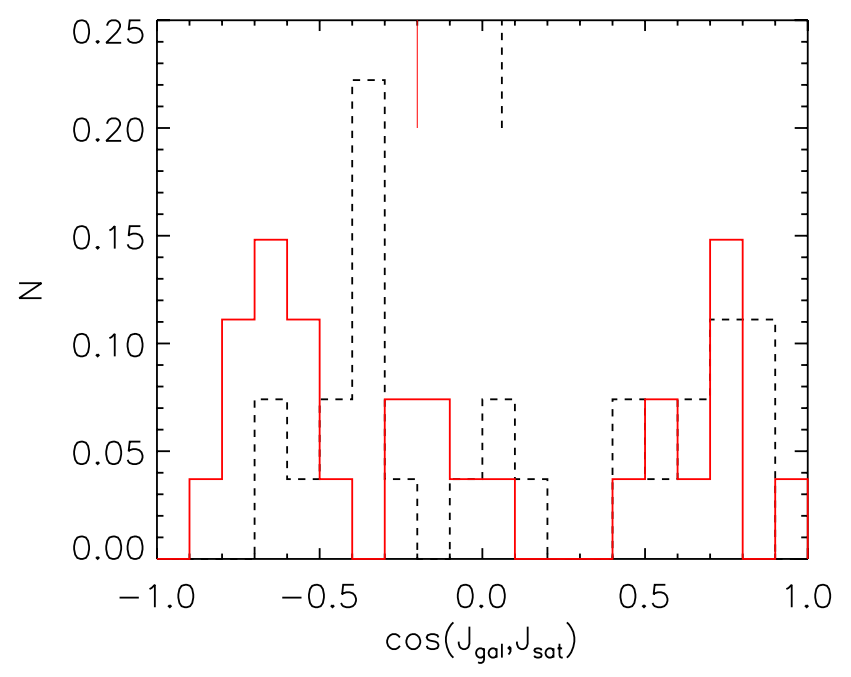

Fig. 7. Cosines between the angular momentum of the progenitor galaxies and that of the accreted satellites, $\cos \left(J_{\text {gal }}, J_{\text {sat }}\right)$. Mergers experienced for simulated galaxies with $D / T_{z=0}>0.5$ (dashed black lines) and with $D / T_{z=0}<0.5$ (solid red lines) are shown separately. The short lines denote the median values for both subsamples.

To explore this idea, we plot the distribution of $\cos \left(J_{\text {gal }}, J_{\text {sat }}\right)$ for all mergers experienced by progenitor systems of the analysed galaxies grouped according to their final D/T. As shown in Fig. 7, low D/T galaxies tend to experience more misaligned encounters (i.e. negative $\cos \left(J_{\mathrm{gal}}, J_{\mathrm{sat}}\right)$ values $)$. However, it should be kept in mind that a larger statistics is needed to confirm this result. The median $\cos \left(J_{\mathrm{gal}}, J_{\mathrm{sat}}\right)$ for galaxies with small disc components is -0.2 , while those with large disc components have 0.06 . Hence, simulated galaxies that were able to reach to $z=0$ with larger discs tend to have experienced more aligned encounters. An average positive alignment of the in-falling structure with the progenitor during the evolutionary history correlates with the growth of the disc, in agreement with previous results (Scannapieco et al. 2009; Sales et al. 2012; Martig et al. 2012).

We also explored the role of the relative masses of the accreted systems, $M_{\mathrm{gal}} / M_{\mathrm{sat}}$ because major mergers are commonly assumed to be responsible of the spheroidal formation. We built up the histograms of $M_{\mathrm{gal}} / M_{\mathrm{sat}}$ for the two subsamples. As can be seen from Fig. 8, low D/T galaxies have a higher probability to have experienced more massive mergers with a median $\left\langle M_{\text {gal }} / M_{\text {sat }}\right\rangle \sim 5$. For galaxies with high $\mathrm{D} / \mathrm{T}$ ratios, the median decreases to $\left\langle M_{\mathrm{gal}} / M_{\mathrm{sat}}\right\rangle \sim 8$. We have checked in both cases that the distributions are statistically different using a Kolmogorov-Smirnov test, which yielded $k_{\mathrm{KS}} \sim 0.07$. 


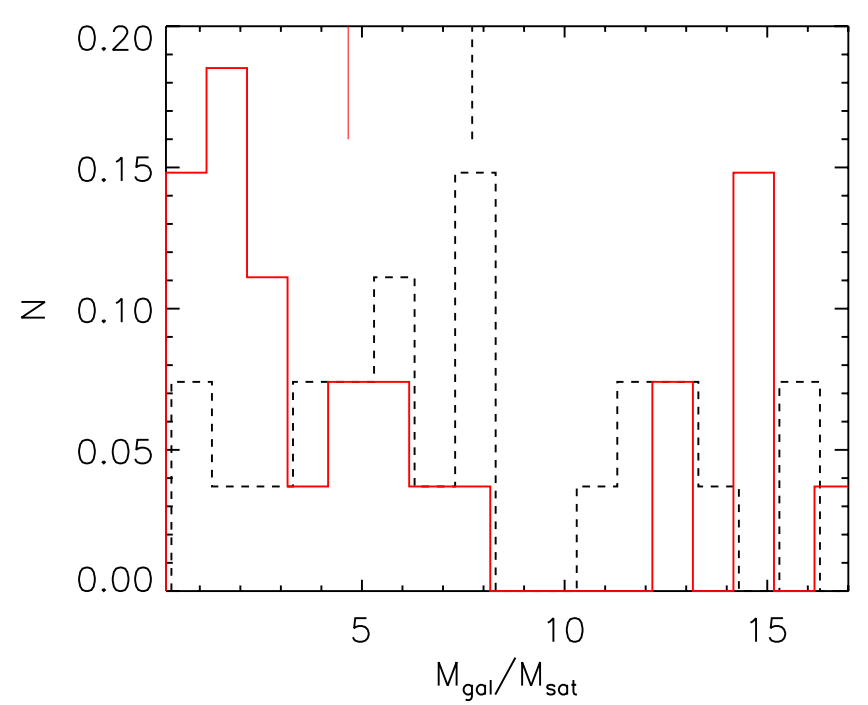

Fig. 8. Progenitor galaxy-to-satellite mass ratio of the 15 chosen galaxies with $D / T_{z=0}>0.5$ (black lines) and with $D / T_{z=0}<0.5$ (red). Median values for both subsamples are shown as short lines.

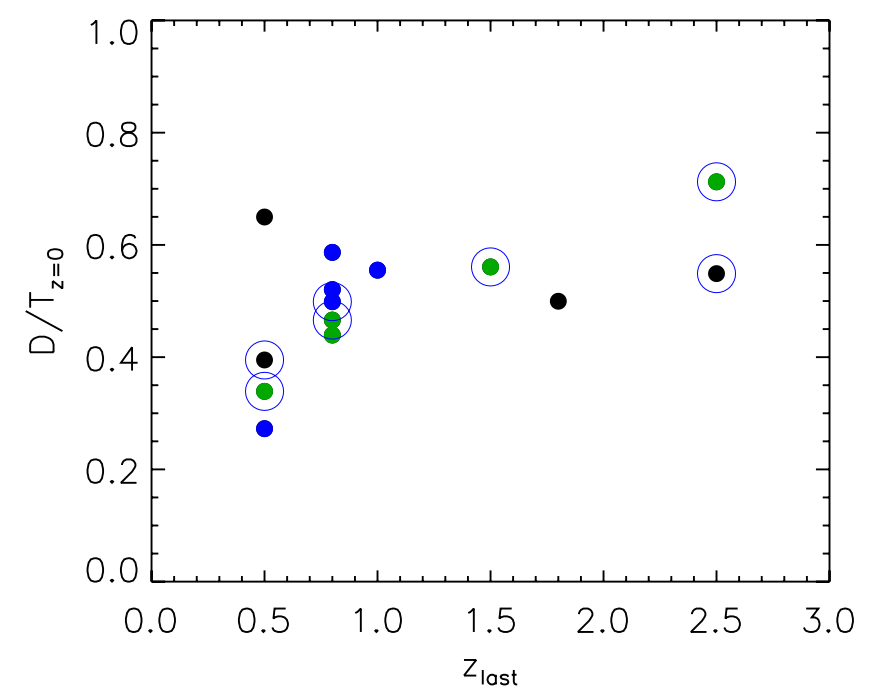

Fig. 9. D/T ratio as a function of the redshift of the last merger. Aligned (misaligned) accretion is shown as blue (green) symbols. Mergers with relative masses larger than 1:6 are enclosed by large circles.

Finally, we estimated that the median redshift for the last merger episode is $z_{\text {last }} \sim 1.4$ for the 15 chosen galaxies with $D / T>0.5$, while for those with $D / T<0.5$, the median is $z_{\text {last }} \sim 0.9$. Hence, even though the 15 chosen galaxies have no major merger for $z<2.5$, there is still a trend for those with larger discs at $z=0$ to experience a quieter evolution in recent epochs. In Fig. 9, we plot D/T as a function of the redshift of the last merger. We included mergers with a mass ratio higher than 1:6 in circles as a reference value to distinguish minor from very small accretions. Mergers that are aligned (misaligned) are shown in blue (green). Systems with destroyed discs tend to experience late mergers, which are, on average, more massive than 1:6. A turn-fork behaviour is suggested from $z_{\text {last }} \sim 1$ related to characteristics of the merger events, which needs to be analysed with a larger sample of galaxies.

\section{Conclusions}

We analysed a sample of 15 galaxies with virial masses between $\sim 3 \times 10^{10}$ to $\sim 3 \times 10^{11}$ in a $\Lambda$-CDM scenario. They all have a quiescent assembly history in the sense that none of them have experienced a major mergers $(1: 3)$ since $z \sim 2.5$. They inhabit typical field regions of the Universe. Nevertheless, they reach redshift zero exhibiting different morphologies. The analysed galaxy sample was resolved with more than 20000 particles within the galaxy radius.

Our results can be summarized as follows:

- We found the disc components to be formed by conserving the specific angular momentum, as expected from the classic disc formation model, so that they determine a relation $j_{\mathrm{d}} \propto m_{\mathrm{d}}^{\alpha}$ with $\alpha \sim 1$, where $j_{\mathrm{d}}$ is the ratio between the specific angular momentum of the galaxy disc and that of the dark matter halo, while $m_{\mathrm{d}}$ is the ratio for the corresponding masses (Mo et al. 1998). The gaseous discs tend to show slightly steeper relation because of action of SN feedback, which determines more extended gas distributions.

We detected that the simulated central spheroids, even though formed from low angular momentum material, determine a clear correlation with the angular momentum of the dark matter haloes. For this simulation, we found $j_{\mathrm{s}} \propto m_{\mathrm{s}}^{\alpha}$ with $\alpha \sim 0.7$. The correlation is noisier than for the disc components, but it is statistically significant. The 15 chosen galaxies follow the mean trends remarkably well. This correlation might explain recent observational results on the mass-size relation reported by Kravtsov (2013). We postpone a deeper discussion on this point to a forthcoming paper where different SN feedback parameters will also be considered.

When all baryons were included, the relation approached $j_{\text {baryons }} \propto m_{\text {baryons }}^{\alpha}$ with $\alpha \sim 2$, in agreement with the results of Sales et al. (2012). The change in the slope is produced by the combined effect of lost and gain of angular momentum due to exchange between components during violent events or by the action of galactic winds (see also Sales et al. 2012).

- The analysis of the formation history of the galaxies that are dominated by the central spheroid or the disc components at $z=0$ show a very complex path. The alignment of the accreted satellites and the relative mass are important for the fate of the discs. Aligned satellites are associated either with a negligible impact or with an increase of the disc mass with respect to the spheroidal mass. During the evolutionary path of the simulated galaxies, we detected a trend for the systems with larger disc components to have experienced, on average, more aligned and less massive mergers than the rest. We therefore confirm results from previous works, which used different numerical codes and SN feedback models (Sales et al. 2012; Martig et al. 2012). Minor mergers can also contribute significantly to the evolution of the galactic morphology depending on their relative alignment with respect to the total angular momentum of the main galaxy.

- At least for this simulation, we found that the final morphology of these systems is determined by $z \sim 1$, on average. It can be affected by posterior events but, on average, their morphology tends to be dominated by previous processes. Gas accretion is required to build up the discs, but our simulation showed that galaxies that reach higher $\mathrm{D} / \mathrm{T}$ ratios are those that reach $z \sim 1$ already with a large disc component. 
Our results show that minor mergers might play a role in determinating the galaxy morphology by contributing to the formation of the disc components if they are aligned, or to the central spheroid, if they are misaligned, with respect to the main disc galaxy. This result extends previous findings (Naab et al. 2009) by showing the role played by the alignment of the minor systems in the formation of each dynamical component from $z \sim 2$. We detected for the first time that the simulated central spheroids formed from material which, although it has low angular momentum content, conserves a relation to that of the dark matter haloes, which might be relevant for understanding the mass-size evolution of galaxies. We expect to go beyond this analysis and achieve a deeper understanding of the processes that determine the morphology with forthcoming simulations with higher resolution and improved physics.

Acknowledgements. We thank the referee for useful comments that helped to clarify the manuscript. This work was partially supported by PICT 2011-0959 from ANPCyT, PIP 2009-0305 and PIP 2012-0396 awarded by CONICET from Argentina. PBT is thankful for support from the Millennium Institute of Astrophysics (MAS) and the Regular Grant UNAB 2014. Simulations were run in the Fenix cluster of the Numerical Astrophysics Group at the Institute for Astronomy and Space Physics.

\section{References}

Agertz, O., Teyssier, R., \& Moore, B. 2011, MNRAS, 410, 1391

Artale, M. C., Pellizza, L. J., Tissera, P. B., \& Mirabel, I. F. 2013, in IAU Symp. 290, eds. C. M. Zhang, T. Belloni, M. Méndez, \& S. N. Zhang, 183

Aumer, M., White, S. D. M., Naab, T., \& Scannapieco, C. 2013, MNRAS, 434, 3142

Barnes, J. E., \& Hernquist, L. 1996, ApJ, 471, 115

Bournaud, F., Perret, V., Renaud, F., et al. 2014, ApJ, 780, 57

Brook, C. B., Governato, F., Roškar, R., et al. 2011, MNRAS, 415, 1051

De Rossi, M. E., Tissera, P. B., \& Pedrosa, S. E. 2010, A\&A, 519, A89

De Rossi, M. E., Tissera, P. B., \& Pedrosa, S. E. 2012, A\&A, 546, A52

De Rossi, M. E., Avila-Reese, V., Tissera, P. B., González-Samaniego, A., \& Pedrosa, S. E. 2013, MNRAS, 435, 2736

Dekel, A., Sari, R., \& Ceverino, D. 2009, ApJ, 703, 785

Diemand, J., Kuhlen, M., Madau, P., et al. 2008, Nature, 454, 735

Domínguez-Tenreiro, R., Tissera, P. B., \& Sáiz, A. 1998, ApJ, 508, L123

Fall, S. M., \& Efstathiou, G. 1980, MNRAS, 193, 189

Governato, F., Zolotov, A., Pontzen, A., et al. 2012, MNRAS, 422, 1231

Guedes, J., Callegari, S., Madau, P., \& Mayer, L. 2011, ApJ, 742, 76

Hopkins, P. F., Bundy, K., Croton, D., et al. 2010, ApJ, 715, 202

Kereš, D., Katz, N., Weinberg, D. H., \& Davé, R. 2005, MNRAS, 363, 2
Khochfar, S., \& Silk, J. 2006, ApJ, 648, L21

Kravtsov, A. V. 2013, ApJ, 764, L31

Laurikainen, E., Salo, H., Buta, R., \& Knapen, J. H. 2007, MNRAS, 381, 401

MacArthur, L. A., Courteau, S., Bell, E., \& Holtzman, J. A. 2004, ApJS, 152, 175

Martig, M., Bournaud, F., Croton, D. J., Dekel, A., \& Teyssier, R. 2012, ApJ, 756,26

Mihos, J. C. 2004, in Clusters of Galaxies: Probes of Cosmological Structure and Galaxy Evolution (Cambridge University Press), 277

Mo, H. J., Mao, S., \& White, S. D. M. 1998, MNRAS, 295, 319

Mosconi, M. B., Tissera, P. B., Lambas, D. G., \& Cora, S. A. 2001, MNRAS, 325,34

Moster, B. P., Naab, T., \& White, S. D. M. 2013, MNRAS, 428, 3121

Naab, T., Johansson, P. H., \& Ostriker, J. P. 2009, ApJ, 699, L178

Okamoto, T., Eke, V. R., Frenk, C. S., \& Jenkins, A. 2005, MNRAS, 363, 1299

Pedrosa, S., Tissera, P. B., \& Scannapieco, C. 2010, MNRAS, 402, 776

Pérez, E., Cid Fernandes, R., González Delgado, R. M., et al. 2013, ApJ, 764, L1

Perez, J., Valenzuela, O., Tissera, P. B., \& Michel-Dansac, L. 2013, MNRAS, 436, 259

Power, C., James, G., Combet, C., \& Wynn, G. 2013, ApJ, 764, 76

Reyes, R., Mandelbaum, R., Gunn, J. E., Pizagno, J., \& Lackner, C. N. 2011 , MNRAS, 417, 2347

Reyes, R., Mandelbaum, R., Gunn, J. E., et al. 2012, MNRAS, 425, 2610

Sales, L. V., Navarro, J. F., Theuns, T., et al. 2012, MNRAS, 423, 1544

Scannapieco, C., Tissera, P. B., White, S. D. M., \& Springel, V. 2005, MNRAS, 364,552

Scannapieco, C., Tissera, P. B., White, S. D. M., \& Springel, V. 2006, MNRAS, 371,1125

Scannapieco, C., Tissera, P. B., White, S. D. M., \& Springel, V. 2008, MNRAS, 389, 1137

Scannapieco, C., White, S. D. M., Springel, V., \& Tissera, P. B. 2009, MNRAS, 396, 696

Sérsic, J. L. 1963, Boletin de la Asociacion Argentina de Astronomia, 6, 41

Springel, V. 2005, MNRAS, 364, 1105

Springel, V., \& Hernquist, L. 2003, MNRAS, 339, 289

Springel, V., Yoshida, N., \& White, S. D. M. 2001, New Astron., 6, 79

Stinson, G. S., Brook, C., Prochaska, J. X., et al. 2012, MNRAS, 425, 1270

Teyssier, R. 2002, A\&A, 385, 337

Thielemann, F.-K., Nomoto, K., \& Hashimoto, M. 1993, in Origin and Evolution of the Elements, eds. N. Prantzos, E. Vangioni-Flam, \& M. Casse, 297

Tissera, P. B. 2000, ApJ, 534, 636

Tissera, P. B., White, S. D. M., \& Scannapieco, C. 2012, MNRAS, 420, 255

Tissera, P. B., Scannapieco, C., Beers, T. C., \& Carollo, D. 2013, MNRAS, 432, 3391

Toomre, A., \& Toomre, J. 1972, ApJ, 178, 623

Torrey, P., Vogelsberger, M., Genel, S., et al. 2014, MNRAS, 438, 1985

Weinzirl, T., Jogee, S., Khochfar, S., Burkert, A., \& Kormendy, J. 2009, ApJ, 696, 411

Woosley, S. E., \& Weaver, T. A. 1995, ApJS, 101, 181 